\title{
Kinetic Modeling and Mathematical Analysis Indicate That Acute Phase Gene Expression in Hep 3B Cells Is Regulated by Both Transcriptional and Posttranscriptional Mechanisms
}

\author{
Shun-Lin Jiang, * David Samols, * Debra Rzewnicki, ${ }^{\S}$ Stephen S. Macintyre, ${ }^{\S}$ Isaac Greber, ${ }^{\ddagger}$ Jean Sipe," \\ and Irving Kushner\$ \\ ${ }^{*}$ Department of Biochemistry and ${ }^{\ddagger}$ Department of Mechanical and Aerospace Engineering, Case Western Reserve University, Cleveland, \\ Ohio 44106; 'Department of Medicine, MetroHealth Medical Center, Cleveland, Ohio 44109; and "Department of Biochemistry, \\ Boston University School of Medicine, Boston, Massachusetts 02118
}

\begin{abstract}
To evaluate the possible role of posttranscriptional mechanisms in the acute phase response, we determined the kinetics of transcription (by nuclear run-on assay) and mRNA accumulation of five human acute phase genes in Hep 3B cells incubated with conditioned medium from LPS-stimulated monocytes. Increase in mRNA accumulation was comparable to increase in transcription rate for fibrinogen- $\alpha$ and $\alpha-1$ protease inhibitor, suggesting largely transcriptional regulation. In contrast, mRNA accumulation was about 10-20-fold greater than transcriptional increase for serum amyloid $A, C 3$, and factor $B$, suggesting participation of posttranscriptional mechanisms. Since finding a disparity between the magnitudes of increase in mRNA and transcription does not definitively establish involvement of posttranscriptional mechanisms, we subjected our data to modeling studies and dynamic mathematical analysis to evaluate this possibility more rigorously. In modeling studies, accumulation curves resembling those observed for these three mRNAs could be generated from the nuclear run-on results only if posttranscriptional regulation was assumed. Dynamic mathematical analysis of relative transcription rates and relative mRNA abundance also strongly supported participation of posttranscriptional mechanisms. These observations suggest that posttranscriptional regulation plays a substantial role in induction of some, but not all acute phase proteins. (J. Clin. Invest. 1995. 95:1253-1261.) Key words: cytokine - serum amyloid $A$ - complement factor $B$ - interleukin-6 $\cdot$ mRNA stability
\end{abstract}

\section{Introduction}

The acute phase response is characterized by a complex set of changes in the concentrations of many plasma proteins, reflecting reorchestration of the pattern of plasma protein gene expression by hepatocytes. A number of inflammation-associated cytokines and hormones have been found to be capable of participating in induction of these changes (1-5). Although transcriptional regulation has been shown to play a major role

Address correspondence to Irving Kushner, MD, Metro Health Medical Center, 2500 MetroHealth Drive, Cleveland, OH 441091998.

Received for publication 3 June 1994 and in revised form 6 September 1994.

J. Clin. Invest.

(C) The American Society for Clinical Investigation, Inc.

0021-9738/95/03/1253/09 \$2.00

Volume 95, March 1995, 1253-1261 in induction of all positive acute phase genes studied thus far, both in vivo and in vitro studies have demonstrated that changes in specific mRNA abundance are not always accompanied by comparable increases in the magnitude of transcription (6-13), suggesting a role for posttranscriptional mechanisms.

The finding of an increase in mRNA levels without a change in transcription is generally accepted as evidence of posttranscriptional control, but the interpretation is more difficult when some increase in transcription is induced but a substantially greater increase in mRNA levels is found. Although this finding is frequently taken to indicate participation of posttranscriptional mechanisms, such conclusions may not always be justified for several reasons. First, when initially low and hence somewhat uncertain transcription rates and mRNA levels are used to calculate the magnitude of increase, substantial errors can result. Second, estimation of transcription rates by nuclear run-on analysis does not yield precise results; a severalfold range of error is not unexpected, rendering interpretation of differences between increases in mRNA and transcription difficult. Third, measurement of increase in transcription and mRNA levels at the same time point may not provide an accurate picture of relative changes in these two parameters, since about five half-lives elapse after the change in transcription rate before a new steady state is reached (14). Only in the case of an mRNA that is rapidly degraded would both transcription rates and mRNA levels be expected to reach their peaks at about the same time. Finally, true steady-state equilibria may not occur during an experiment, since transcription rates, mRNA degradation, and mRNA levels may all be changing continuously over the course of the study.

To evaluate critically the role of cytokine-induced posttranscriptional mechanisms in regulation of human acute phase changes, we determined the effect of continuous exposure to conditioned medium $(\mathrm{CM})^{1}$ from LPS-stimulated human monocytes on the kinetics of transcription and mRNA accumulation of serum amyloid A (SAA), complement factors B ( fB) and $\mathrm{C} 3$, fibrinogen- $\alpha$ ( $\alpha$-fib), and $\alpha$-1 protease inhibitor ( $\alpha$-PI) in Hep 3B cells. To ensure that maximal changes in transcription and mRNA abundance were determined, we performed studies over a period long enough to include peak levels of both transcription and mRNA. To minimize the possible confounding effect of errors resulting from low baseline values of transcription and mRNA, we subjected our data to rigorous mathematical analysis in which neither initial transcription rates nor initial mRNA levels were used in the calculations. Both this approach

1. Abbreviations used in this paper: $\alpha$-fib, fibrinogen- $\alpha$; $\alpha$-PI, $\alpha-1$ protease inhibitor; $\mathrm{CM}$, conditioned medium; fB, complement factor B; SAA, serum amyloid $\mathrm{A}$. 
and kinetic modeling, which also applied quantitative approaches (15) to analysis of our data, were found to support substantial involvement of posttranscriptional mechanisms in induction of SAA, $\mathrm{FB}$, and $\mathrm{C} 3$.

\section{Methods}

CM was prepared from LPS-stimulated human monocytes as previously described (16). Hep 3B cells in RPMI 1640 medium were incubated with $20 \%$ CM by our standard methods with a change of medium, including fresh CM, after each 24-h period. Specific mRNA levels were determined by Northern blot analysis, and relative transcription rates were determined by nuclear run-on assays from monolayers derived from the same cells.

For the nuclear run-on assays $(17,18)$, nuclei from cells in four $100-\mathrm{mm}$ dishes were prepared by lysing washed cells in $10 \mathrm{mM}$ Tris, $\mathrm{pH} 7.4,10 \mathrm{mM} \mathrm{NaCl}, 3 \mathrm{mM} \mathrm{MgCl} 2,0.5 \% \mathrm{NP}-40$ and centrifuging. The resulting nuclei were suspended in $50 \mathrm{mM}$ Tris, $\mathrm{pH} 8.3,40 \%$ glycerol, $5 \mathrm{mM} \mathrm{MnCl}, 0.1 \mathrm{mM}$ EDTA and then frozen in aliquots at $-70^{\circ} \mathrm{C}$. For in vitro transcription assays, $2 \times 10^{7}$ to $4 \times 10^{7}$ nuclei in $90 \mu \mathrm{l}$ were diluted to a final volume of $200 \mu \mathrm{l}$ in buffer so that the final concentrations were $50 \mathrm{mM}$ HEPES, $112 \mu \mathrm{g} / \mathrm{ml}$ creatinine kinase, 2.25 $\mathrm{mg} / \mathrm{ml}$ creatinine phosphate, $4 \mathrm{mM}$ DTT, $0.5 \mathrm{mM}$ CTP, $0.5 \mathrm{mM}$ GTP, $1 \mathrm{mM}$ ATP, and $200 \mu \mathrm{Ci}$ of $\left[{ }^{32} \mathrm{P}\right] \mathrm{UTP}$. Extension of RNA chains that had been initiated at the time of nuclear isolation was continued (run on) by incubating the reaction mixture at $26^{\circ} \mathrm{C}$ for $45 \mathrm{~min}$ before digestion with RNase-free DNase I, proteinase $\mathrm{K}$ and RNA purification by phenol extraction. For hybridization, 1-5 $\mu \mathrm{g}$ of denatured plasmid DNA was loaded into each well of a nitrocellulose filter-containing dot blot manifold, baked at $80^{\circ} \mathrm{C}$ for $2 \mathrm{~h}$, and hybridized with $10^{7} \mathrm{cpm}$ run-on RNA. Hybridization was performed in $10 \mathrm{mM}$ TES, pH 7.4, $10 \mathrm{mM}$ EDTA, $0.2 \% \mathrm{SDS}, 0.3 \mathrm{M} \mathrm{NaCl}$ at $65^{\circ} \mathrm{C}$ for $40 \mathrm{~h}$. Filters were washed with $2 \times$ SSC at $65^{\circ} \mathrm{C}$ for $2 \mathrm{~h}$ and with $20 \mu \mathrm{g} / \mathrm{ml} \mathrm{RNase} \mathrm{A} \mathrm{at} 37^{\circ} \mathrm{C}$ for $30 \mathrm{~min}$. Autoradiographic signals after the hybridization were quantitated by densitometry with a PhosphorImager (Molecular Dynamics, Sunnyvale, CA) and expressed as ratios to the constitutively expressed gene glyceraldehyde-3-phosphate dehydrogenase (GAPDH) (19).

As probes, we used cDNAs for SAA, pA10, courtesy of J. Sipe, Boston, MA (20-22); $\alpha$-fib, courtesy of G. Crabtree, Stanford University, Palo Alto, CA (23); $\alpha$-PI, p8 $\alpha 1 \mathrm{pp} 9$, courtesy of D. Perlmutter, Washington University, St. Louis, MO (24); GAPDH, pHlcGAp3, courtesy of R. Wu, Cornell University, New York (25); C3, C3AL1, courtesy of D. Perlmutter (26); fB, a 1-kb subclone from clone pGEM.Bf, courtesy of D. Perlmutter and H. Colten, Washington University, St. Louis, MO (27).

In initial experiments, we noted a significant signal in baseline nuclear run-on assays of $\mathrm{fB}$, when $\mathrm{fB}$ mRNA was undetectable, that did not increase $6 \mathrm{~h}$ after exposure to cytokines. These findings raised the possibility that the apparent signal found on nuclear run-on assay might be an artifact due to transcription originating outside the gene. To investigate further the nature of the baseline signal in the $\mathrm{fB}$ nuclear run-on assay, we used a plasmid representing a nonrepetitive genomic fragment derived from the 5 ' flanking region of the $\mathrm{fB}$ gene. We also controlled for possible symmetric transcription during the run-on assay by using a single-stranded DNA probe generated by subcloning our existing cDNA into an M13 vector in an orientation such that the RNA complementary strand was produced. An experiment using unstimulated Hep 3B cells revealed no detectable transcription with either the promoter probes or the single-stranded probe. This finding indicated that the elevated $\mathrm{fB}$ nuclear run-on signal observed with our double-stranded cDNA probe was an artifact due to symmetric transcription extending into the fB gene. We concluded that the single-stranded probe provided the truest measure of $\mathrm{fB}$ transcription in our assay system. Accordingly, for later experiments the $\mathrm{fB}$ subclone was inserted into M13MP18, and a single-stranded DNA was prepared with a sequence complementary to $\mathrm{fB}$ mRNA. Using this subclone as the $\mathrm{fB}$ probe, an appropriately low baseline transcription rate was found that rose sharply during incu-

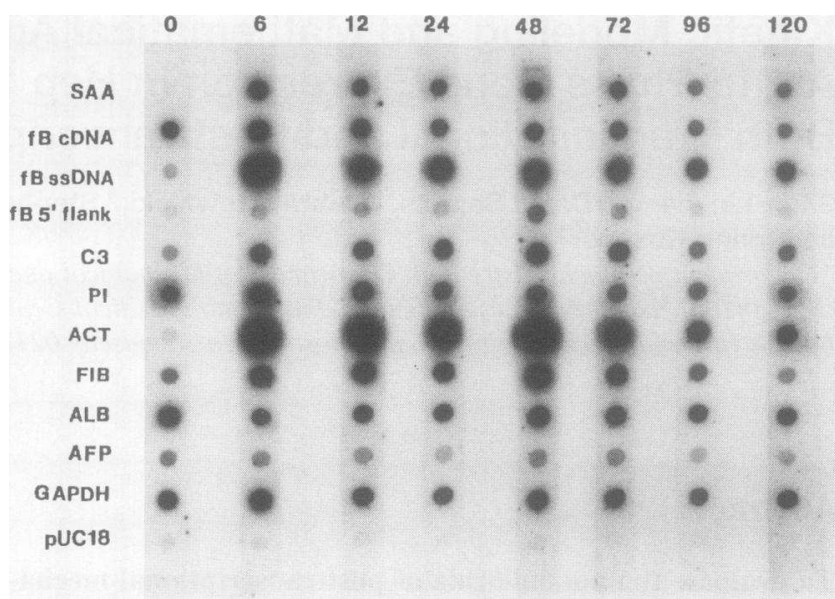

Figure 1. Nuclear run-on assay of acute phase protein genes in Hep 3B cells exposed to CM. 1-5 $\mu$ g of plasmid DNA for each gene indicated was loaded onto a nitrocellulose filter through a dot blot apparatus. cDNAs for each gene except $\mathrm{fB}$ were used. For $\mathrm{fB}$, DNA isolated from the 5' flanking region of the gene and single-stranded DNA complementary to AB mRNA were included. ACT, AFP, and ALB determinations, shown in this figure, are not reported in this manuscript. The filter was cut into strips and hybridized with ${ }^{32} \mathrm{P}$-labeled RNA derived from nuclei from cells exposed to $\mathrm{CM}$ for the indicated times. The figure shows a representative autoradiograph of an experiment extending over $120 \mathrm{~h}$ of CM exposure. The autoradiograph was scanned in a densitometer for quantitative analysis.

bation with CM (Fig. 1). There was no reason to suspect comparable artifacts for any of the other probes used.

Levels of specific RNA were determined by Northern blot analysis of total RNA. RNA samples (15-25 $\mu \mathrm{g}$ ) were heat denatured for $5 \mathrm{~min}$ at $65^{\circ} \mathrm{C}$ in $2 \mathrm{M}$ formaldehyde and fractionated on $0.9 \%$ agarose gels containing $1 \mathrm{M}$ formaldehyde. RNA was transferred to a membrane (GeneScreen, Du Pont, Wilmington, DE) filter that was baked at $80^{\circ} \mathrm{C}$ for $2 \mathrm{~h}$. Each cDNA probe described was labeled by random priming in the presence of $\left[{ }^{32} \mathrm{P}\right] \mathrm{dCTP}$ and hybridized $\left(10^{6} \mathrm{cpm}\right)$; the filter was washed with $2 \times \mathrm{SSC}, 0.1 \%$ SDS at $25^{\circ} \mathrm{C}$ for $15 \mathrm{~min}$ and with $0.2 \times$ SSC, $0.1 \%$ SDS at $65^{\circ} \mathrm{C}$ for $1 \mathrm{~h}$. Hybridized counts were stripped from the filter by heating in $\mathrm{H}_{2} \mathrm{O}$ for $15 \mathrm{~min}$ at $85^{\circ} \mathrm{C}$ before hybridization with the next labeled probe. The amounts of RNA in each lane were normalized to the final hybridization of the filters with a GAPDH probe. Quantitation by densitometry on PhosphorImager was performed as previously described. As a control for cell viability during the $120-\mathrm{h}$ culture period, RNA was isolated from Hep 3B cells maintained in serum-free RPMI1640 medium in parallel with CM-treated cells and analyzed by Northern blots for levels of $\alpha$-PI, albumin, and GAPDH mRNA. These levels were found to be unchanged relative to 18S rRNA during the culture period, indicating that the cells remain viable during this extended period of time without serum.

For secreted protein assays, medium from each sample collection or medium change was saved and used to measure the accumulated amount of each protein at 24-h intervals. Rocket immunoelectrophoresis was used to measure fB, C3, and $\alpha$-PI accumulated in medium. SAA levels were determined using a specific ELISA (28).

\section{Results}

Kinetic studies were performed over 22,72 , and $120 \mathrm{~h}$ of incubation of Hep 3B cells with CM. The temporal patterns of response observed were comparable in all experiments, although peaks of both transcription and mRNA accumulation were achieved at slightly later time points in the 120 -h experiment (shown in Table I), than in the 72-h experiment. Fig. 1 
Table I. Kinetics of Relative mRNA Levels and Transcription Rates during Incubation with CM

\begin{tabular}{|c|c|c|c|c|c|c|c|c|}
\hline \multirow[b]{2}{*}{ Gene } & \multicolumn{8}{|c|}{ Incubation } \\
\hline & $0 \mathrm{~h}$ & $6 \mathrm{~h}$ & $12 \mathrm{~h}$ & $24 \mathrm{~h}$ & $48 \mathrm{~h}$ & $72 \mathrm{~h}$ & $96 \mathrm{~h}$ & $120 \mathrm{~h}$ \\
\hline \multicolumn{9}{|c|}{ mRNA abundance } \\
\hline SAA & $<0.02$ & 0.5 & 1.6 & 3.5 & 8.6 & 10.0 & 10.1 & 7.2 \\
\hline $\mathrm{fB}$ & $<0.05$ & 0.7 & 2.2 & 4.3 & 7.5 & 8.5 & 7.6 & 5.2 \\
\hline $\mathrm{C} 3$ & $<0.05$ & 0.5 & 1.3 & 3.0 & 5.5 & 6.8 & 5.9 & 4.5 \\
\hline$\alpha$-PI & 2.3 & 2.1 & 2.6 & 3.1 & 3.6 & 3.8 & 3.4 & 3.2 \\
\hline$\alpha$-fib & 0.7 & 1.6 & 2.2 & 2.6 & 3.8 & 3.2 & 2.7 & 1.2 \\
\hline \multicolumn{9}{|l|}{ Nuclear run-on } \\
\hline SAA & 0.05 & 0.8 & 0.9 & 1.2 & 1.0 & 1.0 & 0.9 & 0.6 \\
\hline fB (dsDNA) & 0.06 & 0.9 & 1.2 & 1.5 & 0.7 & 0.6 & 0.8 & 0.4 \\
\hline $\mathrm{C} 3$ & 0.2 & 0.6 & 0.9 & 1.5 & 1.4 & 1.1 & 0.9 & 0.6 \\
\hline$\alpha$-PI & 0.7 & 0.8 & 1.2 & 1.5 & 1.0 & 0.8 & 0.1 & 0.7 \\
\hline$\alpha$-fib & 0.3 & 0.7 & 1.4 & 1.3 & 2.1 & 1.1 & 0.8 & 0.2 \\
\hline
\end{tabular}

Data are expressed in densitometric units (ratio of gene of interest to GAPDH). dsDNA, double-stranded DNA.

illustrates a nuclear run-on assay, and Fig. 2 shows a representative Northern blot of $\mathrm{fB}$ over $120 \mathrm{~h}$ of $\mathrm{CM}$ exposure. To estimate baseline values of mRNA for SAA, $\mathrm{FB}$, and $\mathrm{C} 3$, which were undetectable, serial dilutions of RNA extracted at $6 \mathrm{~h}$ were loaded on the same agarose gel, and the sensitivity of our Northern blot assay for each of these mRNA species was determined (see Fig. 2 for dilutions with $\mathrm{fB}$ probe). We assumed that the steady-state levels of these mRNA species in unstimulated cells could be no more than the lower limit of sensitivity thus determined and used these values in Table $I$ and in calculations of the magnitude of change.

Fig. 3 illustrates kinetics of change in mRNA levels and transcription rates during a 120 -h exposure to $\mathrm{CM}$, expressed as fold increase compared with observed or calculated baseline values. Rapidly rising transcription rates were observed for all acute phase genes during incubation with $\mathrm{CM}$; peak values were attained at $24 \mathrm{~h}$ with the exception of $\alpha$-fib, which attained a

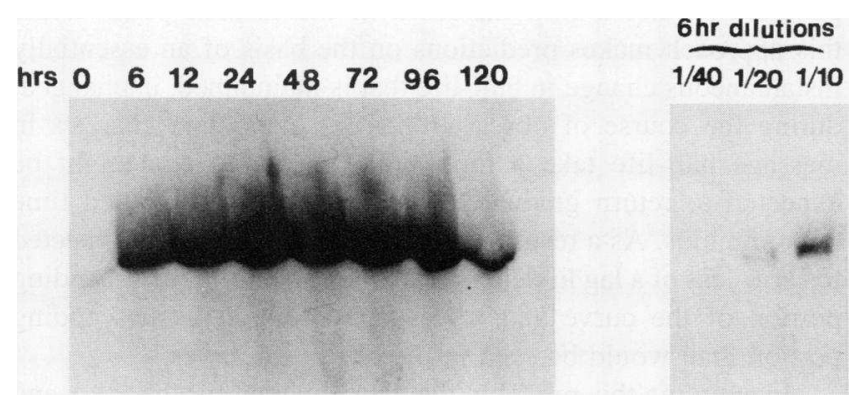

Figure 2. Northern blot analysis of $\mathrm{fB}$ mRNA in Hep 3B cells exposed to CM. Total RNA from Hep 3B cells exposed to CM for the indicated times was isolated and fractionated by formaldehyde-agarose gel electrophoresis before transfer to GeneScreen membranes and hybridization as described in Methods. The figure shows a representative autoradiograph using a $\mathrm{fB}$ cDNA probe of an experiment extending over 120 $h$ of $C M$ exposure. The right-hand panel is another portion of the same filter showing the fB Northern blot signal of the 6-h RNA sample after the dilutions indicated to determine the limit of detection for RNAs in which only a weak signal was evident in the unstimulated RNA population. The autoradiograph was scanned in a densitometer for quantitative analysis before removal of hybridized RNA and counter-hybridization with another probe. peak at $48 \mathrm{~h}$. Table II depicts calculated ratios between increases in mRNA and in transcription, as well as the time lags between peak transcription and peak or plateau mRNA levels for all genes studied. Two kinetic patterns emerged from these data. $\mathrm{SAA}, \mathrm{fB}$, and C3 mRNA levels increased $~ 10-20$-fold more than did transcription rates. We concluded that posttranscriptional events might play an important role in regulation of these genes by CM. In contrast, increases in transcription rates and mRNA levels were roughly comparable for both $\alpha$-fib and $\alpha$ PI. For these genes, we concluded that transcriptional changes could largely account for the changes in mRNA induced by $\mathrm{CM}$.

SAA, $\mathrm{fB}$, and C3 mRNA levels continued to rise after transcription rates began to fall, with a consequent substantial lag between peak transcription and mRNA levels, suggesting that these mRNAs are relatively long lived (14). In contrast, for $\alpha$ PI and $\alpha$-fib, no significant time lag was seen between peaks of transcription and mRNA accumulation, suggesting that these mRNAs are relatively unstable.

Secretion of SAA, $\mathrm{fB}, \mathrm{C} 3$, and $\alpha$-PI into medium was essentially linear during a 120-h incubation with CM (Fig. 4). Comparable linear secretion of these proteins was found in the 72$h$ experiment. These findings attested to the continued viability of our cells during the entire period of study.

Modeling and mathematical analysis of data. To test our tentative conclusion that these data suggested substantial involvement of posttranscriptional mechanisms in induction of SAA, fB, and C3, we used first a curve-fitting, modeling approach and then a rigorous mathematical analysis of the data. The modeling method makes the simplifying assumption that any posttranscriptional effects are exerted at the level of mRNA degradation, which is then used as a surrogate for posttranscriptional regulation in general. This method allows the initial halflife of an mRNA to be varied and then predicts the accumulated mRNA levels (based on observed changes in transcription rate) under conditions in which the mRNA half-life either remains constant or changes after stimulus. The predicted mRNA accumulation curves are then compared with the observed data from Northern blots. In each case, the initial transcription rate is assigned a value of 1 unit, and the units expected to be present in the initial, steady-state mRNA pool are determined for each 
SAA

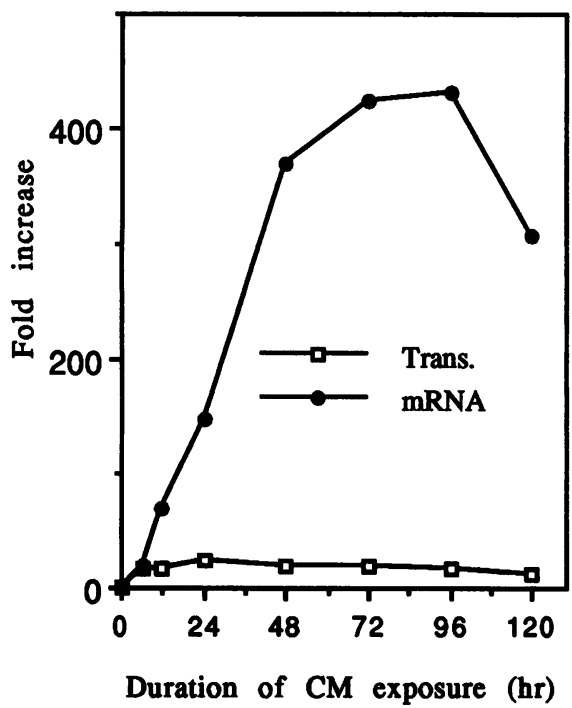

Factor B

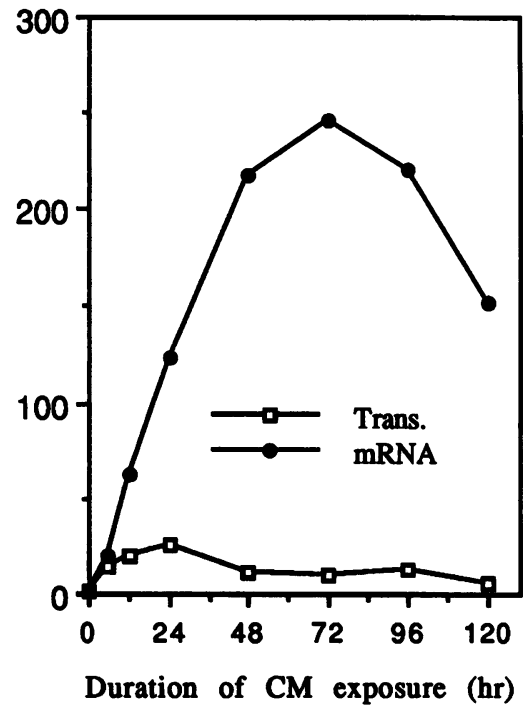

C3

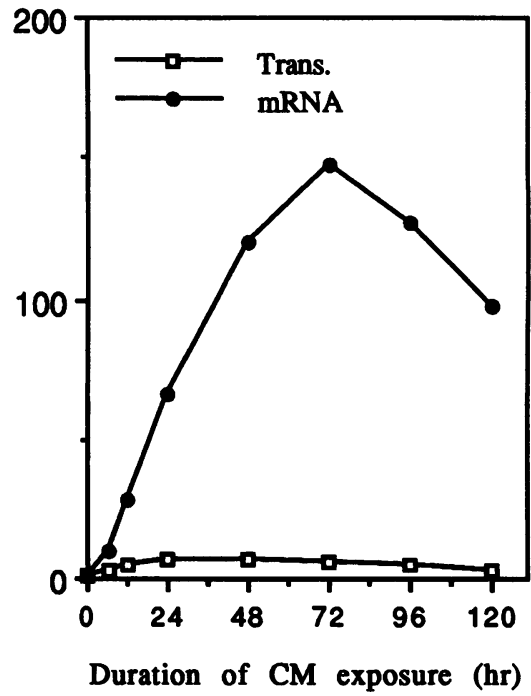

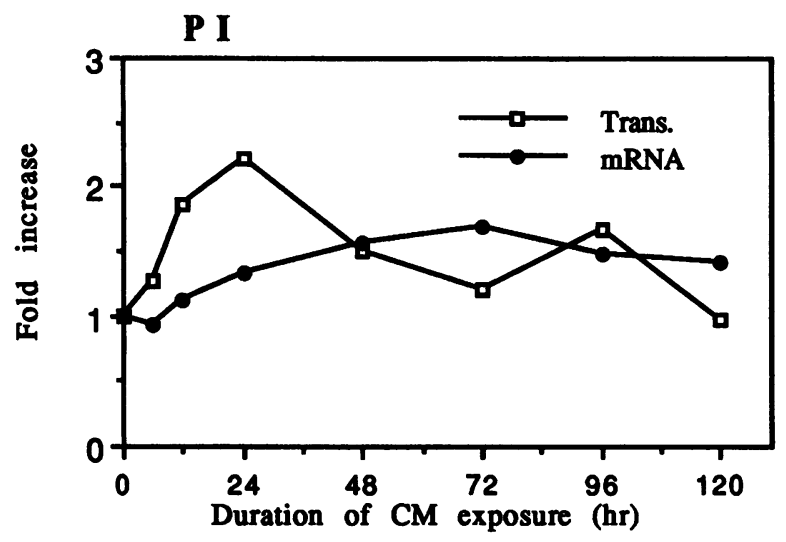

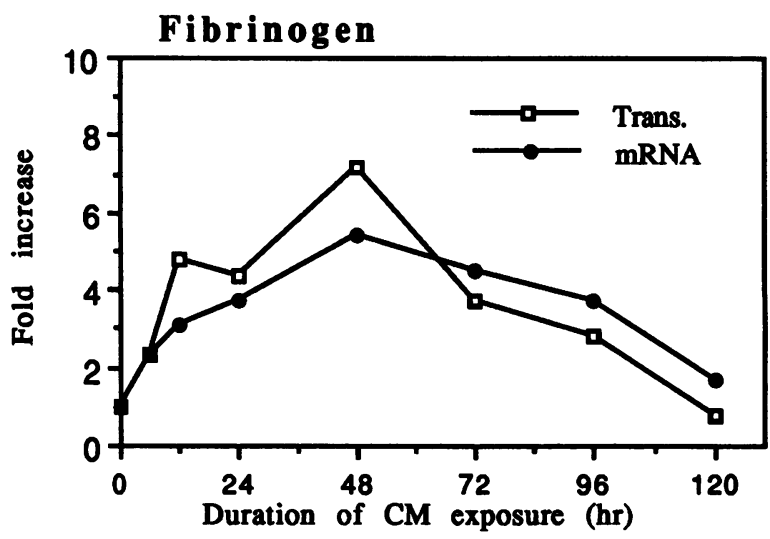

Figure 3. Kinetics of change in transcription as reflected by nuclear run-on assays (Trans) and mRNA abundance ( $m R N A)$ of five acute phase genes in Hep 3B cells during a 120-h incubation with CM. Points are represented as fold increase over baseline values set equal to 1 .

assigned initial half-life as follows: for each time interval, the size of the mRNA pool is the sum of the number of units at the beginning of the interval, plus the input to the pool, less the exit from the pool as determined by the assigned half-life. The size of the initial mRNA pool (in arbitrary units) is then used as the denominator to predict relative fold increases in the predicted pool values. The same procedure is then applied to intervals after stimulus, in which relative transcription rates are varied, as determined from the nuclear run-on data, in order to

Table II. Transcriptional and mRNA Changes during 120-h Incubation with $C M$

\begin{tabular}{lcc}
\hline & $\begin{array}{c}\text { Fold increase in mRNA/ } \\
\text { fold increase in transcription }\end{array}$ & $\begin{array}{c}\text { Time lag (h) between } \\
\text { transcription peak and } \\
\text { mRNA plateau or peak }\end{array}$ \\
\hline$\alpha$-PI & $1.7 / 2.2=0.8$ & 24 \\
$\alpha$-fib & $5.4 / 7.2=0.75$ & 0 \\
SAA & $>430 / 24=>17.9$ & 48 \\
fB & $>240 / 25=>9.6$ & 48 \\
C3 & $>147 / 7.3=>20.1$ & 48 \\
\hline
\end{tabular}

generate predicted fold increases in the mRNA pool. While this approach makes predictions on the basis of an essentially instantaneous change in half-life that is maintained, unchanged, during the course of observation, it is likely that changes in message half-life take a finite time to evolve and might be expected to return gradually to baseline with continued time after stimulus. As a result, the model curves would be expected to show less of a lag in rising mRNA levels during the ascending portion of the curve and a less rapid fall in the descending portion than would be seen in the observed curves.

In applying this model to the data obtained for the SAA and $\mathrm{fB}$ genes, none of a wide range of assigned half-lives accurately predicted the accumulation curves if the half-lives were kept constant (Fig. $5 \mathrm{~A}$ ). Short half-lives were found to generate the necessary level of mRNA accumulation, but plateaus were achieved too early compared with the observed data. Assigning longer half-lives generated curves that reached plateau levels at times consistent with those observed, but at mRNA levels that were much lower than those observed experimentally. In contrast, for each of these genes, a relatively narrow range of initial half-lives was found to approximate more closely the observed levels of mRNA accumulation, if the initial half-lives were allowed to increase after stimulus (Fig. $5 B$ ). The best fit 


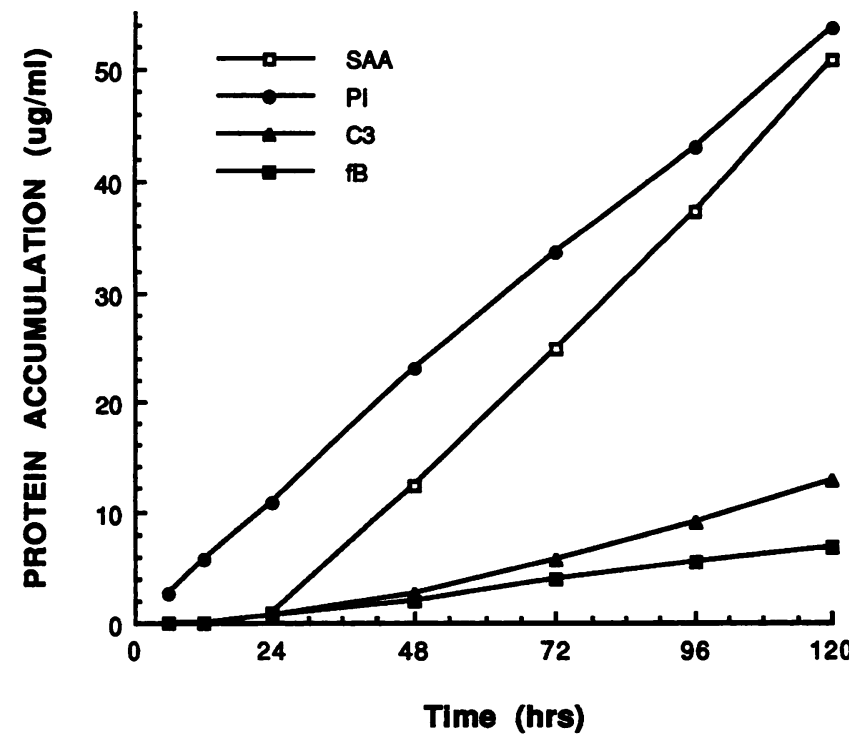

Figure 4. Secretion of plasma proteins into medium. Aliquots of medium were collected after $6,12,24,48,72,96$, and $120 \mathrm{~h}$, and the concentration of plasma proteins was determined. Results are plotted as cumulative amounts.

for SAA was observed with an assigned initial half-life of 5 $6 \mathrm{~h}$ and a 5-7-fold increase after stimulus; the best fit for $\mathrm{fB}$ was observed with an assumed initial half-life of 7-8 $\mathrm{h}$ and a 4-6-fold increase after stimulus. In contrast, the model indicated that the observed accumulation of $\alpha$-fib could be accounted for on the basis of an initial half-life of $\sim 12 \mathrm{~h}$, with no change after stimulus (Fig. $5 C$ ).

In addition to modeling, the observed data were subjected to dynamic mathematical analysis to estimate changes in fractional degradation of mRNA over the course of the experiments. As with the modeling approach, mRNA degradation was used as a surrogate for all posttranscriptional effects. Since only relative, rather than absolute, values of mRNA abundance and transcription rates can be obtained from the experiments, all values are referred to reference values (described in the following discussion). The notation is as follows: $p$ is the transcription rate at any time; $p_{\mathrm{r}}$ is the reference transcription rate; $x$ is the abundance of any species of mRNA at any time; $x_{\mathrm{r}}$ is the reference abundance; and $r$ is the degradation rate at any time.

The amount existing at any time (the abundance) minus the initial amount is equal to the amount produced minus the amount degraded. Symbolically, this can be written as

$x-x_{0}=\int_{0}^{t} p \mathrm{~d} t-\int_{0}^{t} r \mathrm{~d} t$.

The experiments determine $p / p_{\mathrm{r}}$ and $x / x_{\mathrm{r}}$. Rewriting Eq. 1 in terms of the measurable values, one has

$\frac{x}{p_{\mathrm{r}} t_{\mathrm{r}}}\left(\frac{x}{\mathrm{x}_{\mathrm{r}}}-\frac{x_{0}}{x_{\mathrm{r}}}\right)=\int_{0}^{t / t_{\mathrm{r}}}\left(\frac{p}{p_{\mathrm{r}}}-\frac{r}{p_{\mathrm{r}}}\right) \mathrm{d}\left(\frac{t}{t_{\mathrm{r}}}\right)$,

where $t_{\mathrm{r}}$ is a reference time.

Average values during the period of the study have been selected as the reference values, among other reasons because these minimize any errors due to uncertainties in determination of baseline values. If one uses average values as the reference values and the total time as the reference time, then the front factor on the left-hand side of Eq. $2, x_{\mathrm{r}} / p_{\mathrm{r}} t_{\mathrm{r}}$, is the average mRNA abundance over the course of the experiment divided by the total amount transcribed. We will call this the "abundance ratio" and denote it by $C$. Observe that the abundance ratio is not determined by the experiments and cannot be known unless one can obtain absolute rather than relative values. Typically $x_{0} / x_{\mathrm{r}}$ is much less than unity, so that $C$ can take on values in the range 0 to 1 .

By applying Eq. 2 at different times, one can obtain the normalized accumulated degradation to that time, for specified values of the abundance ratio. The explicit formula, a rewriting of Eq. 2, is

$\int_{0}^{t / t_{\mathrm{r}}} \frac{r}{p_{\mathrm{r}}} \mathrm{d}\left(\frac{t}{t_{\mathrm{r}}}\right)=\int_{0}^{t / t_{\mathrm{r}}} \frac{p}{p_{\mathrm{r}}} \mathrm{d}\left(\frac{t}{t_{\mathrm{r}}}\right)-C\left(\frac{x}{x_{\mathrm{r}}}-\frac{x_{0}}{x_{0}}\right)$.

When Eq. 3 is applied to a time interval rather than to the total time, one can deduce the average degradation rate for that time interval. One also can obtain the corresponding normalized degradation rate per unit abundance by dividing the degradation rate by the scaled, normalized abundance at the middle of the time interval; the explicit formula is

$\frac{1}{x} \frac{\mathrm{d} x_{\mathrm{d}}}{\mathrm{d}\left(t / t_{\mathrm{r}}\right)}=\frac{1}{C} \frac{r / p_{\mathrm{r}}}{x / x_{\mathrm{r}}}=R / C$,

where $x_{\mathrm{d}}$ is the amount degraded. For convenience, in scales of the graphs we will use $R$ as a nondimensional measure of the rate per unit abundance and will call $R$ the fractional degradation rate.

If desired, the degradation half-life at any time can be determined from $R$ and $C$ as follows:

$t_{1 / 2}=\left[t_{\mathrm{r}} \ln (2)\right](C / R)$.

Note that $t_{1 / 2}$ is not a constant.

One can arbitrarily assign abundance ratios within allowable limits, to determine relative changes in fractional degradation rates with time. The allowable abundance ratios range from the largest value consistent with the requirement that the degradation at any time cannot be $<0$ to values approaching 0 , reflecting high degrees of degradation. It is likely that the complexity of the RNA being transcribed and measured by nuclear run-on assay is far greater than that of the mature RNA species measured by Northern blots. Given the known rapid turnover rates of the highly complex nuclear pre-mRNA, it is likely that the lower values of $C$ more closely reflect the biological state. This is particularly true when one appreciates that the denominator of $C$ represents total transcription over the course of the experiment, independent of degradation; it must be a relatively large quantity, and $C$, of necessity, must be small.

As shown in Fig. 6, at all assumed values of $C$, ranging from 0.25 to 0.001 , fractional degradation rates of SAA demonstrated the same behavior: a rapid fall after the first 6-h period, with persistently low values thereafter. This observation, that fractional degradation rates fall (and half-lives increase) with cytokine exposure, confirms the conclusion that posttranscriptional mechanisms participate in SAA mRNA induction by $\mathrm{CM}$. For $\mathrm{fB}$ and $\mathrm{C} 3$, similar curves were seen with assigned $C$ values of 0.1 and lower, but not at values of 0.25 and 0.15 , respectively (Fig. 6). These observations are consistent with, but do not establish the conclusion that posttranscriptional mechanisms participate in induction of $\mathrm{fB}$ and $\mathrm{C} 3$. This conclusion is, however, more strongly supported if the lower values of $C$ are regarded as valid, consistent with the known rapid turnover of 
A

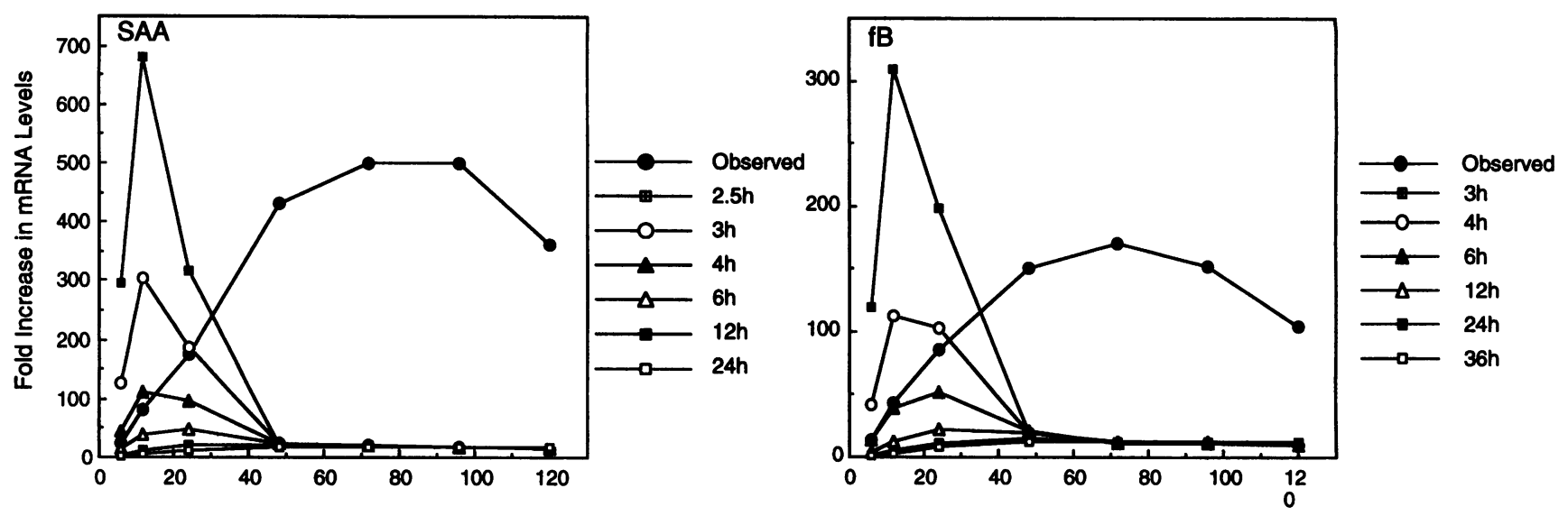

Time After Induction (h)

B
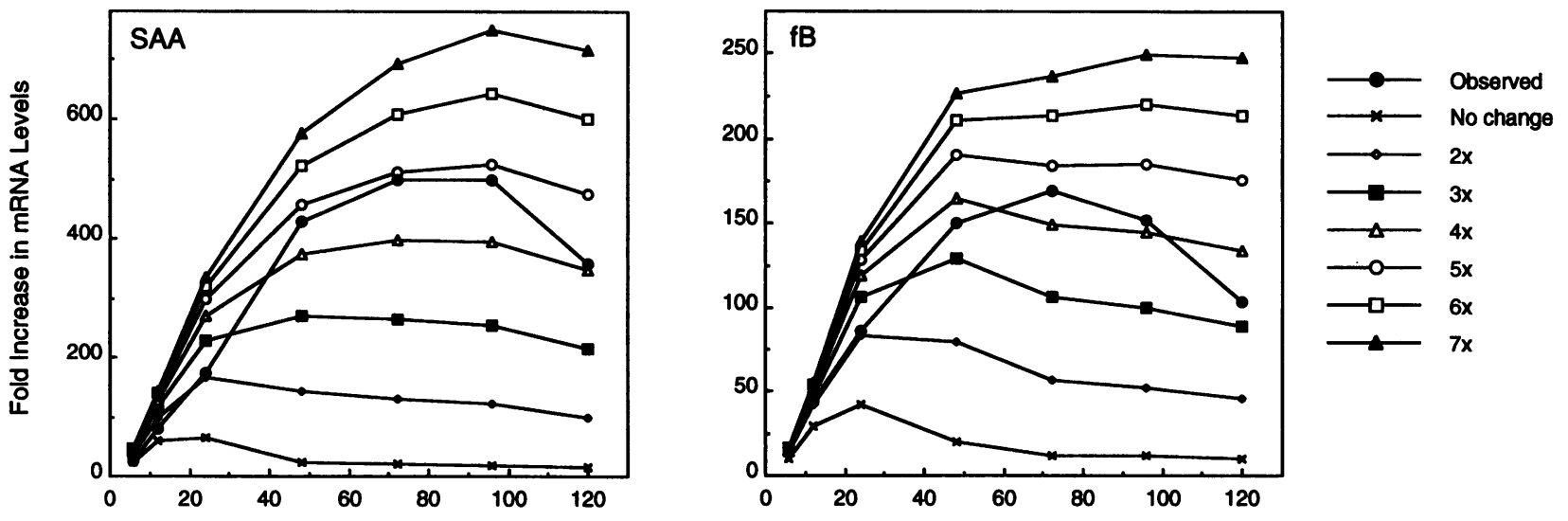

Time After Induction (h)

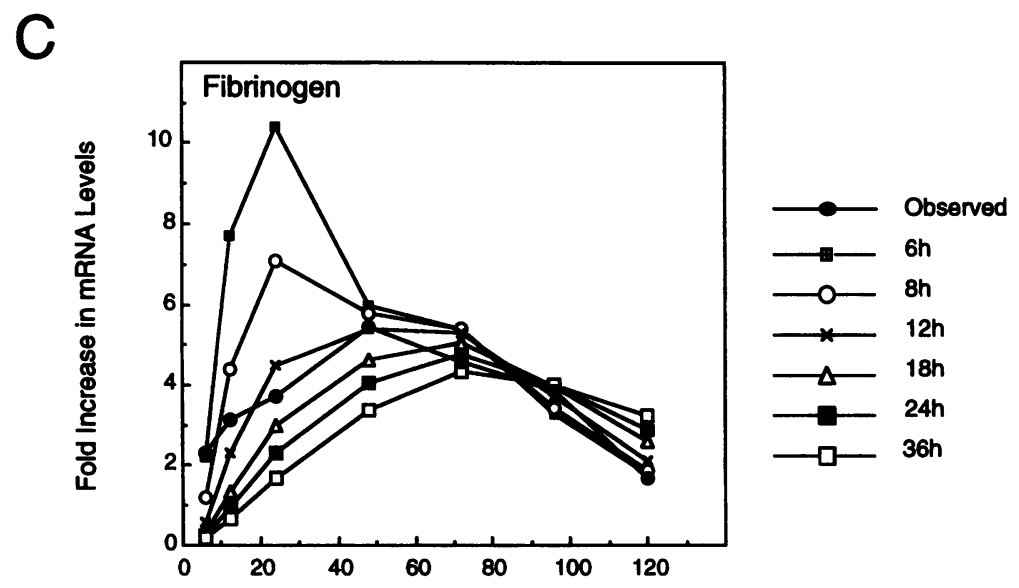

Time After Induction (h)

Figure 5. Mathematically predicted mRNA levels at intervals after stimulus, assuming different half-lives of mRNA, based on modeling. (A) Predicted mRNA values for SAA and $\mathrm{fB}$ at different assigned values of $\mathrm{mRNA}$ half-lives, held constant. Experimentally observed changes in mRNA accumulation are indicated by the closed circles. $(B)$ Predicted mRNA values for SAA and $\mathrm{fB}$, assuming initial half-lives of $5 \mathrm{~h}$ for SAA, $7 \mathrm{~h}$ for $\mathrm{fB}$, and increases in half-life as indicated. (C) Predicted mRNA values for $\alpha$-fib at different assigned values of mRNA half-lives, held constant. 

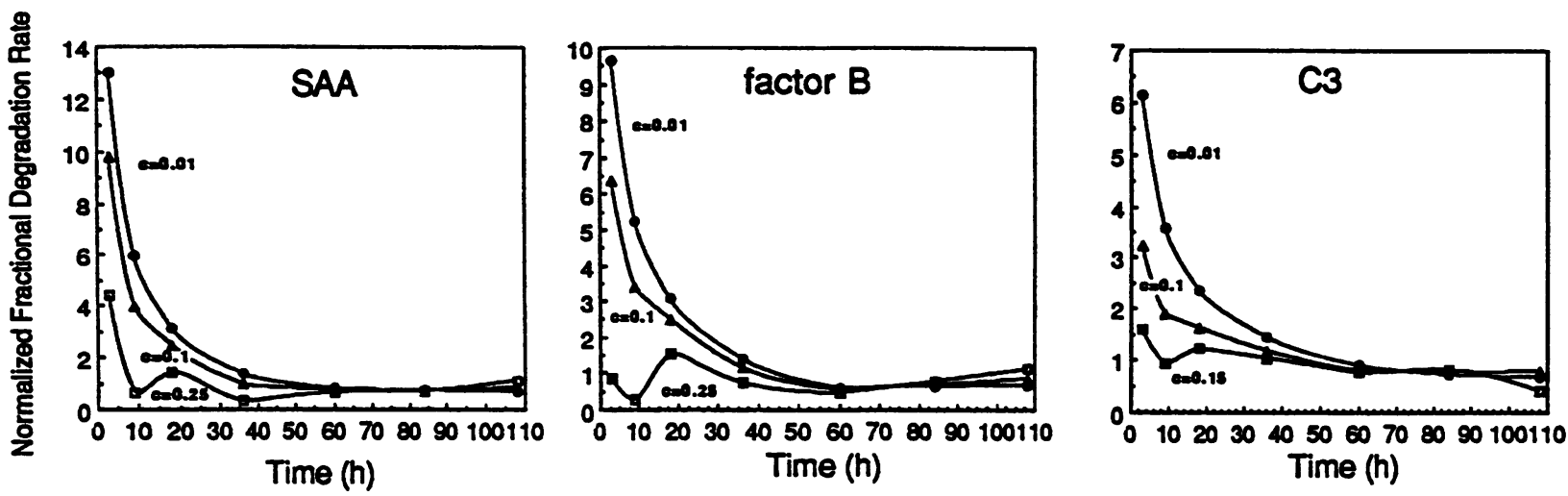

Figure 6. Calculated normalized fractional degradation rates of SAA, fB, and C3 mRNAs for different assigned values of abundance ratios $(C)$ during incubation of Hep 3B cells.

nuclear pre-mRNA. In contrast, for $\alpha$-PI and $\alpha$-fib, relatively minor changes in fractional degradation rates were found at all assigned values of $C$, and substantial decreases in fractional degradation rates were not seen. These observations suggest that posttranscriptional mechanisms do not play major roles in induction of these mRNAs.

It should be reemphasized that regulation of either transcript processing or transport of mRNA from the nucleus to the cytoplasm could be consistent with these data as well, since an initial assumption that mRNA degradation was the only effective posttranscriptional mechanism was made for these analyses.

\section{Discussion}

These kinetic studies were undertaken to help determine whether changes in acute phase mRNA levels induced by $\mathrm{CM}$ in Hep 3B cells could be fully explained by effects of transcription alone, or whether posttranscriptional mechanisms need to be invoked to explain the changes observed. CM caused increased transcription of each of the positive acute phase genes we studied, indicating that the changes in mRNA were at least partially attributable to transcriptional regulation. However, the observation that the magnitudes of increase in mRNA for SAA, $\mathrm{FB}$, and C3 were substantially greater than the magnitudes of increase in transcription suggested that posttranscriptional mechanisms contributed to the changes in expression induced in these genes by CM. To evaluate this possibility rigorously, we undertook two mathematically based, quantitative approaches to test the validity of this conclusion.

Our curve-fitting, modeling approach supported the conclusion that posttranscriptional mechanisms must be invoked to explain the observed effects on mRNA of SAA, fB, and C3, since no constant half-life values were capable of predicting the observed mRNA accumulation curves. In addition, making the working assumption that these posttranscriptional mechanisms influenced mRNA degradation rather than affected nuclear processing of primary transcripts or export of mature mRNA, they permitted crude estimates of the magnitudes of effect on mRNA stability required to account for the observed findings.

A significant concern in the interpretation of these data was the need to use relatively small transcription rates found in unstimulated cells as denominators for determination of the magnitude of increase in transcription. Were these values falsely high, the true magnitude of transcription rate increase would, in fact, be higher than we calculated and closer to the changes seen with mRNA. Similarly, low, imperfectly quantitated initial mRNA levels might lead to false calculation of the magnitude of increase. To avoid the necessity of relying on initial transcription and mRNA values for mathematical analysis, we used abundance ratios (which represented average - rather than initial-mRNA abundance) divided by total transcription over the period of the experiment (which also avoided use of initial transcription rate values). Thus, neither numerator nor denominator was subject to the sources of error that might result from the use of very small baseline values. Using this approach, dynamic mathematical analyses indicated that SAA induction by $\mathrm{CM}$ involved substantial participation of posttranscriptional mechanisms and was strongly supportive of such a conclusion for $\mathrm{fB}$ and $\mathrm{C} 3$. Although intranuclear processing, decay of transcripts, and nucleocytoplasmic export of mRNA might all theoretically serve as posttranscriptional regulatory mechanisms (for review see reference 29 ), kinetic modeling has suggested that such mechanisms would be relatively inefficient in affecting cytoplasmic mRNA concentrations and that the main points of control must be transcriptional processes and regulation of mRNA degradation (14). Thus far, in a few limited studies, relatively modest direct effects of cytokines on stability of acute phase mRNAs in cell lines have been reported (30-32). Studies of some murine SAA genes $(9,12)$ have suggested posttranscriptional control, but effects on mRNA stability could not be demonstrated using the transcriptional inhibitor actinomycin D. However, the observations of Steel et al. (33) that the disappearance rate of SAA mRNA during actinomycin D treatment is substantially slower than its disappearance rate after cytokine induction, which we have independently confirmed, indicate that this method cannot be used for estimation of the stability of SAA mRNA.

We used CM as a potent stimulus to induce acute phase changes since such preparations have repeatedly been found to induce the entire spectrum of these changes in Hep 3B cells $(16,34,35)$. CM preparations consist of a crude and undefined mixture of IL-6 and other inflammation-associated cytokines, cytokine modulators (such as IL-1 receptor antagonist), and endocrine hormones, such as dexamethasone and insulin. The potency of various CM preparations may vary from one experiment to another; greater effects were seen in our 120-h experiment than in our 72-h experiment. Future studies delineating specific mechanisms involved in acute phase induction will require the use of defined cytokines, cytokine modulators, and hormones. In initial studies of this type, we have found that the 
combination of IL- 6 and IL- $1 \beta$ caused a substantially greater increase in mRNA levels than in transcription for SAA, fB, and $\mathrm{C} 3$, but not for $\alpha$-PI, supporting the findings observed with CM (35a).

Because mRNA levels of SAA, $\mathrm{fB}$, and $\mathrm{C} 3$ were undetectable in unstimulated cells, it was necessary to estimate maximal baseline mRNA levels from estimates of the sensitivity of the method. The approach we used, determining the lower limit of detectability of each mRNA by serial dilutions of 6-h samples, yielded the maximal possible value of baseline mRNA levels. It is likely that actual mRNA levels were lower than these values, that the magnitudes of increase we calculated were minimal estimates, and that we thus underestimated the actual magnitude of mRNA increase that occurred. To the extent that this is the case, it would further strengthen the argument for participation of substantial posttranscriptional mechanisms in induction of these three genes.

In summary, these studies suggest that posttranscriptional mechanisms participate in induction of several acute phase genes by $\mathrm{CM}$. The increasing number of eukaryotic genes found to be regulated at the level of mRNA stability and the multiplicity of mechanisms by which this regulation may be accomplished $(36,37)$ suggest that studies of such mechanisms in acute phase genes may prove informative. Future studies should include critical evaluation of the possible effects of acute phaseinducing cytokines on mRNA stability.

\section{Appendix}

\section{Derivation of Formulas}

Analysis of degradation. The abundance, $x$, at any time is the initial abundance, $x_{0}$, plus the amount produced minus the amount degraded. Calling the rate of production $p$ and the rate of degradation $r$, the above statement can be written

$x=x_{0}+\int_{0}^{t} p \mathrm{~d} t-\int_{0}^{t} r \mathrm{~d} t$.

The experiments do not measure abundance or production rates directly; they provide the values relative to the corresponding values of GAPDH. If the GAPDH values are assumed to be constant, then the experiments yield the ratios of abundance and production rate to the corresponding values at a chosen reference time. Symbolically this can be written

$\frac{x}{x_{1}}=\frac{x / x_{\mathrm{G}}}{x_{1} / x_{\mathrm{G}}}$

$\frac{p}{p_{1}}=\frac{p / p_{\mathrm{G}}}{p_{1} / p_{\mathrm{G}}}$

where the subscripts 1 and G denote specified time and GAPDH, respectively.

Rewriting Eq. Al as a formula for the amount of degradation yields

$p_{1} \int_{0}^{t} \frac{r}{p_{1}} \mathrm{~d} t=p_{1} \int_{0}^{t} \frac{p}{p_{1}} \mathrm{~d} t-x_{1}\left(\frac{x}{x_{1}}-\frac{x_{0}}{x_{1}}\right)$.

Instead of using the values of $x$ and $p$ at some specified time as reference values, it is convenient to use the average values as reference values. Denoting the average value by the subscript $r$, the averages can be obtained as follows:

$\frac{x_{\mathrm{r}}}{x_{\mathrm{l}}} t_{\mathrm{r}}=\int_{0}^{t_{\mathrm{r}}} \frac{x}{x_{1}} \mathrm{~d} t$,

$\frac{p_{\mathrm{r}}}{p_{1}} t_{\mathrm{r}}=\int_{0}^{t_{\mathrm{r}}} \frac{p}{p_{1}} \mathrm{~d} t$ where $t_{\mathrm{r}}$ is the total time of the experiment. Then

$\frac{x}{x_{\mathrm{r}}}=\frac{x / x_{1}}{x_{\mathrm{r}} / x_{1}} \quad \frac{p}{p_{\mathrm{r}}}=\frac{p / p_{1}}{p_{\mathrm{r}} / p_{1}}$

Eq. A4 remains valid with the subscript 1 replaced by subscript $r$. It is convenient to perform the integrations Eq. Al using as a variable the time of the observation relative to the total time of the experiment. Then Eq. A4 can be rewritten

$p_{\mathrm{r}} t_{\mathrm{r}} \int_{0}^{t / t_{\mathrm{r}}} \frac{r}{p_{\mathrm{r}}} \mathrm{d}\left(\frac{t}{t_{\mathrm{r}}}\right)=p_{\mathrm{r}} t_{\mathrm{r}} \int_{0}^{t / t_{\mathrm{r}}} \frac{p}{p_{\mathrm{r}}} \mathrm{d}\left(\frac{t}{t_{\mathrm{r}}}\right)-x_{\mathrm{r}}\left(\frac{x}{x_{\mathrm{r}}}-\frac{x_{0}}{x_{\mathrm{r}}}\right)$.

Dividing through by $p_{\mathrm{r}} t_{\mathrm{r}}$ yields

$\int_{0}^{t / t_{\mathrm{r}}} \frac{r}{p_{\mathrm{r}}} \mathrm{d}\left(\frac{t}{t_{\mathrm{r}}}\right)=\int_{0}^{t / t_{\mathrm{r}}} \frac{p}{p_{\mathrm{r}}} \mathrm{d}\left(\frac{t}{t_{\mathrm{r}}}\right)-\frac{x_{\mathrm{r}}}{p_{\mathrm{r}} t_{\mathrm{r}}}\left(\frac{x}{x_{\mathrm{r}}}-\frac{x_{0}}{x_{\mathrm{r}}}\right)$.

Eq. A9 corresponds to Eq. 3 of the main text. For any assigned value of $x_{\mathrm{r}} / p_{\mathrm{r}} t_{\mathrm{r}}$, Eq. A9 yields the total amount degraded from the beginning of the experiment until the specified time, relative to the amount that could be produced at the average production rate. Applying Eq. A9 between two times of observation, $a$ and $b$, yields the average degradation rate over the time interval $t_{\mathrm{b}}-t_{\mathrm{a}}$, in the same nondimensional sense as described above. This can be written

$\frac{r}{p_{\mathrm{r}}}=\frac{\int_{\left(t / t_{\mathrm{r}}\right)_{\mathrm{a}}}^{(t / t)_{\mathrm{r}}} \frac{r}{p_{\mathrm{r}}} \mathrm{d}\left(\frac{t}{t_{\mathrm{r}}}\right)}{\left(\frac{t}{t_{\mathrm{r}}}\right)_{\mathrm{b}}-\left(\frac{t}{t_{\mathrm{r}}}\right)_{\mathrm{a}}}$.

The above-average value of $r / p_{\mathrm{r}}$ should be regarded as the value at the midpoint of the time interval $t_{\mathrm{b}}-t_{\mathrm{a}}$.

The degradation rate per unit abundance $r / x$ is given by

$r / x=\left(\frac{r / p_{\mathrm{r}}}{x / x_{\mathrm{r}}}\right)\left(\frac{p_{\mathrm{r}} t_{\mathrm{r}}}{x_{\mathrm{r}}}\right) \frac{1}{t_{\mathrm{r}}}$

It is convenient to use the nondimensional value, $r t_{\mathrm{r}} / x$ (which we will call $R / C$ ) as a measure of the fractional degradation rate, where $C$ is the abundance ratio $x_{\mathrm{r}} / p_{\mathrm{r}} t_{\mathrm{r}}$. The abundance ratio $C$ is the average mRNA abundance divided by the total amount transcribed. The quantity $R / C$ can be interpreted as the nondimensional derivative, $d / d\left(t / t_{\mathrm{r}}\right)$, of the amount degraded, $x_{\mathrm{d}}$, per unit abundance. Thus one rewrites Eq. A11 as follows:

$\frac{1}{x} \frac{\mathrm{d} x_{\mathrm{d}}}{\mathrm{d}\left(t / t_{\mathrm{r}}\right)}=\frac{p_{\mathrm{r}} t_{\mathrm{r}}}{x_{\mathrm{r}}}\left(\frac{r / p_{\mathrm{r}}}{x / x_{\mathrm{r}}}\right)=\frac{R}{C}$.

Eq. A12 corresponds to Eq. 4 of the main text.

Relationship of degradation with half-life. The half-life at any time is obtained by considering a constant fractional rate of degradation and no production. Then the rate at which the abundance increases is the negative of the degradation rate. This can be written

$-\frac{1}{x} \frac{\mathrm{d} x}{\mathrm{~d} t}=\frac{r}{x}=$ constant $\equiv K$.

Integrating Eq. A13 yields

$\ln \left(x / x_{1}\right)=-K t$.

The half-life is the time such that $x / x_{1}=1 / 2$. Then $K t=\ln (2)$. With $K=r / x$ given by Eq. A11, the half-life is given by

$t_{1 / 2}=t_{\mathrm{r}} \ln (2) / R$,

where $R$ is the nondimensional fractional degradation rate given in Eq. A12.

\section{Acknowledgments}

We are grateful for the outstanding secretarial assistance of Nancy Kessler and Lori Fransen. Appendix prepared by Isaac Greber, Professor of Mechanical \& Aeronautical Engineering. 
This work was supported by National Institutes of Health grant AG-02467.

\section{References}

1. Richards, C. D., T. J. Brown, M. Shoyab, H. Baumann, and J. Gauldie 1992. Recombinant oncostatin M stimulates the production of acute phase proteins in Hep G2 cells and rat primary hepatocytes in vitro. J. Immunol. 148:17311736.

2. Mackiewicz, A., M. K. Ganapathi, D. Schultz, A. Brabenec, J. Weinstein, M. F. Kelley, and I. Kushner. 1990. Transforming growth factor $\beta 1$ regulates production of acute phase proteins. Proc. Natl. Acad. Sci. USA. 87:1491-1495.

3. Schooltink, H., T. Stoyan, E. Roeb, P. C. Heinrich, and S. Rose-John. 1992. Ciliary neurotrophic factor induces acute-phase protein expression in hepatocytes. FEBS (Fed. Eur. Biochem. Soc.) Lett. 314:280-284.

4. Baumann, H., C. Richards, and J. Gauldie. 1987. Interaction among hepatocyte-stimulating factors, interleukin 1 , and glucocorticoids for regulation of acute phase plasma proteins in human hepatoma (Hep G2) cells. J. Immunol. 139:41224128.

5. Campos, S. P., and H. Baumann. 1992. Insulin is a prominent modulator of cytokine-stimulated expression of acute phase plasma protein genes. Mol. Cell. Biol. 12:1789-1797.

6. Birch, H. E., and G. Schreiber. 1986. Transcriptional regulation of plasma protein synthesis during inflammation. J. Biol. Chem. 261:8077-8080.

7. Gehring, M. R., B. R. Shiels, W. Northemann, M. H. L. de Bruijn, C.-C Kan, A. C. Chain, D. J. Noonan, and G. H. Fey. 1987. Sequence of rat liver $\alpha_{2}$ macroglobulin and acute phase control of its messenger RNA. J. Biol. Chem. 262:446-454.

8. Geiger, T., T. Andus, J. Klapproth, H. Northoff, and P. C. Heinrich. 1988. Induction of $\alpha_{1}$-acid glycoprotein by recombinant human interleukin-1 in rat hepatoma cells. J. Biol. Chem. 2612:7141-7146.

9. Lowell, C. A., R. S. Stearman, and J. F. Morrow. 1986. Transcriptional regulation of serum amyloid A gene expression. J. Biol. Chem. 261:8453-8461.

10. Morrone, G., R. Cortese, and V. Sorrentino. 1989. Post-transcriptiona control of negative acute phase genes by transforming growth factor beta. $E M B O$ (Eur. Mol. Biol. Org.) J. 8:3767-3771.

11. Northemann, W., M. Heisig, D. Kunz, and P. C. Heinrich. 1985. Molecular cloning of cDNA sequences for rat $\alpha_{2}$-macroglobulin. J. Biol. Chem. 260:62006205 .

12. Rienhoff, H. Y. J., and M. Groudine. 1988. Regulation of amyloid A gene expression in cultured cells. Mol. Cell. Biol. 8:3710-3716.

13. Shiels, B. R., W. Northemann, M. R. Gehring, and G. H. Fey. 1987. Modified nuclear processing of $\boldsymbol{\alpha}_{1}$-acid-glycoprotein RNA during inflammation. J. Biol. Chem. 262:12826-12831.

14. Hargrove, J. L. 1993. Microcomputer-assisted kinetic modeling of mammalian gene expression. FASEB (Fed. Am. Soc. Exp. Biol.) J. 7:1163-1170.

15. Maddox, J. 1992. Is molecular biology yet a science? Nature (Lond.). 355:201

16. Ganapathi, M. K., D. Schultz, A. Mackiewicz, D. Samols, S. I. Hu, A. Brabenec, S. S. Macintyre, and I. Kushner. 1988. Heterogeneous nature of the acute phase response. Differential regulation of human serum amyloid A, C reactive protein, and other acute phase proteins by cytokines in Hep 3B cells. $J$. Immunol. 141:564-569.

17. Gillaspy, G. E., T. B. Mapstone, D. Samols, and D. A. Goldthwait. 1992 Transcriptional patterns of growth factors and proto-oncogene in human glioblastomas and normal glial cells. Cancer Lett. 65:55-60.

18. Greenberg, M. E., and E. B. Ziff. 1984. Stimulation of 3T3-cells induces transcription of the c-fos proto-oncogene. Nature (Lond.). 311:433-437.

19. Milland, J. A. T., T. Thomas, A. R. Aldred, T. Cole, and G. Schreiber 1990. Gene expression in regenerating and acute phase rat liver. Am. J. Physiol. 2159:G340-G347.
20. Sipe, J. D., H. R. Colten, G. Goldberger, M. D. Edge, B. F. Tack, A. S. Cohen, and A. S. Whitehead. 1985. Human serum amyloid A (SAA): biosynthesis and postsynthetic processing of preSAA and structural variants defined by complementary DNA. Biochemistry. 24:2931-2936.

21. Kluve-Beckerman, B., G. L. Long, and M. D. Benson. 1986. cDNA sequence evidence for polymorphic forms of human serum amyloid A. Biochem. Genet. 24:795.

22. Sipe, J. D., P. Woo, G. Goldberger, A. S. Cohen, and A. S. Whitehead. 1986. Characterization of two distinct serum amyloid A gene products defined by their complementary DNAs. In Amyloidosis. G. G. Glenner, E. Osserman, E. P. Benditt, E. Calkins, A. S. Cohen, and D. Zucker-Franklin, editors. Plenum Publishing Corp., New York. p. 57.

23. Kant, J. A., S. T. Lord, and G. R. Crabtree. 1983. Partial mRNA sequences for human Aa, BB, and gamma fibrinogen chains: evolutionary and functional implications. Proc. Natl. Acad. Sci. USA. 80:3953-3957.

24. Ciliberto, G., L. Dente, and R. Cortese. 1985. Cell-specific expression of a transfected human $\alpha 1$-antitrypsin gene. Cell. 41:537-540.

25. Tso, J. Y., X.-H. Sun, T.-H. Kao, K. S. Reece, and R. Wu. 1985. Isolation and characterization of rat and human glyceraldehyde-3-phosphate dehydrogenase cDNAs: genomic complexity and molecular evolution of the gene. Nucleic Acids Res. 13:2485-2502.

26. Strunk, R. C., A. S. Whitehead, and F. S. Cole. 1985. Pretranslational regulation of the synthesis of the third component of complement in human mononuclear phagocytes by the lipid A portion of lipopolysaccharide. J. Clin. Invest. 76:985-990.

27. Nonaka, M., J. D. Gitlin, and H. R. Colten. 1989. Regulation of human and murine complement: comparison of $5^{\prime}$ structural and functional elements regulating human and murine complement factor B gene expression. Mol. Cell. Biochem. 89:1-14.

28. Sipe, J. D., W. A. Gonnerman, L. D. Loose, G. Knapschaefer, W. J. Xie, and C. Franzblau. 1989. Direct binding enzyme-linked immunosorbent assay (ELISA) for serum amyloid A (SAA). J. Immunol. Methods 125:125-135.

29. Morrow, C. S., J. Chiu, and K. H. Cowan. 1992. Post-transcriptional control of glutathione S-transferase $\alpha$ gene expression in human breast cancer cells. J. Biol. Chem. 267:10544-10550.

30. Lappin, D. F., D. Guc, A. Hill, T. McShane, and K. Whaley. 1992. Effect of interferon-gamma on complement gene expression in different cell types. Biochemistry. 281:437-442.

31. Westerhausen, D. R., Jr., W. E. Hopkins, and J. J. Billadello. 1991. Multiple transforming growth factor- $\beta$-inducible elements regulate expression of the plasminogen activator inhibitor type-1 gene in Hep G2 cells. J. Biol. Chem. 266:1092-1100.

32. Woo, P., M. R. Edbrooke, J. Betts, G. Watson, and P. Francis. 1993. Serum amyloid A gene regulation. In Acute Phase Proteins. Molecular Biology, Biochemistry, and Clinical Applications. A. Mackiewicz, I. Kushner, and H. Baumann, editors. CRC Press, Boca Raton, FL. p. 397-408.

33. Steel, D. M., J. T. Rogers, M. C. DeBeer, F. C. deBeer, and A. S. Whitehead. 1993. Biosynthesis of human acute phase serum amyloid A protein (A-SAA) in vitro: the roles of mRNA accumulation, poly (A) tail shortening and translational efficiency. Biochem. J. 291:701-707.

34. Darlington, G. J., D. R. Wilson, and L. B. Lachman. 1986. Monocyteconditioned medium, interleukin-1, and tumor necrosis factor stimulate the acute phase response in human hepatoma cells in vitro. J. Cell Biol. 103:787-793.

35. Morrone, G., G. Ciliberto, S. Oliviero, R. Arcone, L. Dente, J. Content, and $R$. Cortese. 1988. Recombinant interleukin 6 regulates the transcriptional activation of a set of human acute phase genes. J. Biol. Chem. 263:12554-12558.

35a. Jiang, S.-L., G. Lozanski, D. Samols, and I. Kushner. 1995. Induction of human serum amyloid $A$ in Hep 3B cells by IL-6 and IL- $1 \beta$ involves both transcriptional and posttranscriptional mechanisms. J. Immunol. 154:825-831.

36. Cleveland, D. W., and T. J. Yeo. 1989. Multiple determinants of eukaryotic mRNA stability. New Biologist. 1:121-126.

37. Sachs, A. B. 1993. Messenger RNA degradation in eukaryotes. Cell. $74: 413-421$ 